Filippo Brighina

Giuseppe Salemi

Brigida Fierro

Antonio Gasparro

Giovanni Balletta

Antonina Aloisio

Giovanni Battista La Pegna

Giovanna Randisi

Vincenzo Saporito

Giuseppe Calagna

Filippo Lanaia

Rossana Morana

Published online: 20 July 2005

F. Brighina (凶) • G. Salemi • B. Fierro Dipartimento di Neurologia,

Oftalmologia, Otorinolaringoiatria e Psichiatria, Università di Palermo,

Via G. La Loggia 1, I-90129 Palermo, Italy

e-mail: fbrighina@neuro.unipa.it

Tel.: +39-091-6555108

Fax: +39-091-6555102

A. Gasparro

UO di Neurologia, Ospedale Villa Sofia,

Palermo, Italy

G. Balletta • A. Aloisio

UO di Neurologia, Ospedale Civico,

Palermo, Italy

G.B. La Pegna

UO di Neurologia, ASL n. 2,

Caltanissetta, Italy

G. Randisi

UO di Neurologia, Ospedale S. Elia,

Caltanissetta, Italy

\section{Saporito}

Servizio Neurologia,

Distretto di Bagheria AUSL n. 6,

Bagheria (PA), Italy

G. Calagna

Ospedale Civico di Partitico, ASL n. 6,

Partitico (PA), Italy

F. Lanaia • R. Morana

Dipartimento di Neurologia,

Università di Catania,

Catania, Italy

\title{
A validation study of an Italian version of the ID Migraine: preliminary results
}

\begin{abstract}
Migraine is a highly prevalent and disabling disease that is substantially undiagnosed in primary care. Recently, the ID Migraine, a self-administered questionnaire, was shown to be a valid and reliable screener for migraine in primary care in the USA. To validate an Italian version of the ID Migraine, we planned a multicentric study, evaluating at least 220 patients affected by various form of headache. The responses to the questionnaire were compared with the diagnosis of headache made by a headache specialist blind to the result of the questionnaire. Sensitivity, specificity, and positive and negative predictive values for migraine were calculated. The statistical analysis on 140 patients now examined showed a very good performance of the ID Migraine with high sensitivity: 0.94 (95\% CI: 0.89-0.95), specificity: 0.70 (95\% CI: 0.54-0.86) and positive predictive value: 0.89 (0.82-0.95). If confirmed, these results would establish ID Migraine as a valid screening instrument for migraine in Italian headache patients and warrant further investigation in primary care to assess the validity of this ID screener in Italian population.
\end{abstract}

Key words Migraine • ID migraine • Italian ID migraine $\cdot$ Migraine recognition $\cdot$ Headache centers • Primary care 


\section{Introduction}

Although migraine represents an important cause of temporary disability $[1,2]$, as shown by recent surveys, about $50 \%$ of persons with migraine, even those with disabling headache, have never consulted a physician for the problem [3]. Moreover, only one third of migraine sufferers are currently treated with a prescription drug [4]. The low rates of diagnosis and treatment have several causes, including low medical consultation specifically for headache. Improving recognition of migraine in primary care will increase the rate of successful treatment with effective migraine-specific therapies. Recently, Lipton et al. validated a very brief self-administered questionnaire, consisting of only 3 items, the ID Migraine, for screening of migraine headache in primary care practice (PCP) [5]. ID Migraine was found to be a very good tool for recognition of migraine sufferers, showing very high sensitivity, specificity and positive predictive value (PPV) for migraine headache in a primary care setting.

The aim of the present study was to validate an Italian version of ID Migraine, to be used for migraine screening in Italian headache patients.

\section{Subjects and methods}

We planned a multicentric study involving several headache centres to evaluate at least 220 consecutive patients affected by various forms of headache. The size of the sample to be examined was determined taking into account the prevalence of migraine headaches.

Consecutive headache patients aged $18-65$ years referring to the headache centres involved in the study and reporting at least two headaches in the last three months were eligible for the study; according to the inclusion criteria used by Lipton et al. [5], patients had also to indicate that they had experienced at least a headache that interfered with their lives. Each patient completed an Italian version of the ID Migraine (Fig. 1), previously translated by Pfizer, which also has the copyright of the original ID Migraine. Pfizer authorised the authors to use the Italian version of the ID questionnaire for screening of migraine in an Italian headache population.
Patients gave their informed consent to participate in the study, which was approved by the local ethics committee.

According to Lipton et al. [5], the response to each item was treated as a binary variable with a "no" assigned to responses of "never" or "rarely" and "yes" assigned to responses of "less than half the time" or "half the time or more."

After completing the questionnaire, patients were evaluated by a board-qualified headache specialist blind to the result of the ID Migraine. He performed a complete clinical evaluation including medical history, physical examination, comprehensive neurologic history and examination (including additional diagnostic tests if clinically indicated), and made headache diagnosis according to the criteria of the new classification of the International Headache Society (IHS), which was considered the gold standard [6].

The responses to the items of the questionnaire were then compared with the diagnosis and the validity was assessed calculating sensitivity, specificity, positive (PPV) and negative (NPV) predictive values. Test-retest reliability was evaluated in an independent sample of 20 patients that repeated the questionnaire 2-5 days after, through Kappa coefficient for intraclass correlation.

\section{Results}

We have now evaluated 140 patients (F/M: 98/42, mean age: $38 \pm 12.7$ ) affected by various forms of headache, about $60 \%$ of the sample to be examined. Seventy per cent of them (98 patients; F/M: 71/27; mean age: $36.7 \pm 11.8$ ) were affected by migraine and the remaining $30 \%$ (42 patients; F/M: 28/14; mean age: $36.7 \pm 11.8$ ) by nonmigraine headache. In this last group $67 \%$ had tensive, $10 \%$ cluster and $23 \%$ other headaches. Table 1 shows sensitivity, specificity, positive (PPV) and negative (NPV) predictive values of positive responses to the ID items for the diagnosis of migraine.

Analysis of the single questions showed high values of sensitivity and PPVs for each item (>80\%), disability reaching the highest score in sensitivity 0.98 (0.96-1.01); and photophobia the highest PPV: 0.89 (0.82-0.96). Specificity had generally lower scores; lower scores were observed also in NPV for all items

\section{Questionario ID Migraine:}

Durante gli ultimi tre mesi ha avuto i seguenti disturbi contemporaneamente al mal di testa?

1. Ha avuto nausea o conati di vomito?

sì no

2. Le ha dato fastidio la luce (molto di più di quando non ha mal di testa)?

3. Il mal di testa ha limitato, per almeno un giorno la sua capacita di lavorare, studiare o fare quello che deve? sì

sì 
Table 1 Validity scores of single and combination of two out of the three items of the ID Migraine

\begin{tabular}{lrrrr}
\hline & Sensitivity (CI 95\%) & Specificity (CI 95\%) & PPV (CI 95\%) & NPV (CI 95\%) \\
\hline Nausea & $0.85(0.78-0.92)$ & $0.64(0.47-0.79)$ & $0.86(0.79-0.93)$ & $0.62(0.46-0.78)$ \\
Photophobia & $0.77(0.68-0.86)$ & $0.76(0.59-0.93)$ & $0.89(0.82-0.96)$ & $0.56(0.41-0.71)$ \\
Disability & $0.98(0.96-1.01)$ & $0.52(0.34-0.68)$ & $0.84(0.77-0.91)$ & $0.95(0.85-1.5)$ \\
2 Items positive & $0.94(0.89-0.98)$ & $0.70(0.54-0.86)$ & $0.89(0.82-0.95)$ & $0.83(0.70-0.96)$ \\
\hline
\end{tabular}

$P P V$, positive predictive value; $N P V$, negative predictive value

except disability, which showed the highest NPV value: 0.95 (0.85-1.5). Empirical combinations of the three items of the ID questionnaire found that positive response on any two out of the three items gave an optimal score with sensitivity of 0.94 (95\% CI: 0.89-0.95), specificity of 0.70 (95\% CI: $0.54-0.86)$, PPV of 0.89 (0.82-0.95) and NPV of $0.83(0.70-0.96)$. In the subjects that took the questionnaire twice, test-restest reliability was good (Kappa coefficient: 0.70).

\section{Discussion}

This study reports results on about $60 \%$ of the sample we planned to examine to evaluate the validity of an Italian version of the ID Migraine. Even though preliminary, validity and reliability measures show that the three-item ID Migraine Screener appears to be a valid and reliable screening instrument for migraine headaches also in Italian headache patients. Indeed it had sensitivity of 0.94 (95\% CI: 0.89-0.95), specificity of 0.70 (95\% CI: $0.54-0.86)$, PPV of $0.89(0.82-0.95)$ and NPV of 0.83 $(0.70-0.96)$ for the diagnosis of migraine headaches. These scores indicate an overall good validity level of the Italian version of the ID questionnaire and are also in good agreement with the results by Lipton et al. [5], which found sensitivity of 0.81 (95\% CI, 0.77-0.85), specificity of 0.75 (95\% CI, 0.64-0.84) and PPV of 0.93 (95\% CI, 89.9-95.8) of the ID Migraine screener in a primary care setting in the USA. Compared to this study, in our sample we had higher sensitivity and lower specificity scores. These differences however are slight and not relevant as concerns the validity of the Italian as compared to the English version of the ID Migraine. Moreover, the repetition of the Italian version of the ID Migraine in an independent sample of 20 patients showed a good test-retest reliability with a Kappa coefficient of 0.70 , which is very similar to the value of 0.68 found by Lipton et al. [5].

We used ID Migraine in headache centres, a setting in which a different, higher rate and severity of migraine headache could be expected with respect to PCP. This could have affected measures like NPV and PPV that are critically dependent on the prevalence of the disorder in the sample. Because of these limitations, the validity of our results should be confined, at least as concerns NPV and PPV measures, to the patient population of tertiary headache centres. However, the percentage of migraineurs in our sample $(70 \%)$ is similar to that reported by Lipton et al., who found 332 patients affected by migraine on a total validation sample of 443 patients screened $(75 \%)$. This high prevalence could be due to the following inclusion criteria used by Lipton et al. to favour identification of migraineurs in PCP attendees complaining of headache: two headaches in the previous 3 months and a disabling headache in the past.

In conclusion, preliminary observations on $60 \%$ of the sample we planned to examine showed the Italian version of the ID Migraine as a valid and reliable tool for screening of migraine in Italian headache patients. If confirmed in the total sample, these results would warrant further validation of the ID Migraine screener in PCP. This indeed would establish the Italian ID Migraine as a simple and effective tool to increase recognition of migraineurs also in an Italian population, giving these patients the chance of more specific and effective treatment.

\section{References}

1. Terwindt GM, Ferrari MD, Tijhuis M et al (2000) The impact of migraine on quality of life in the general population: the GEM study. Neurology 55:624-629
2. Hu XH, Markson LE, Lipton RB, Stewart WF, Berger ML (1999) Burden of migraine in the United States: disability and economic costs. Arch Intern Med 159:813-815
3. Lipton RB, Diamond S, Reed M, Diamond M, Stewart WF (2001) Migraine diagnosis and treatment: results from the American Migraine Study II. Headache 41:646-657 
4. Lipton RB, Scher AI, Kolodner K, Liberman J, Steiner TJ, Stewart WF (2002) Migraine in the United States: epidemiology and patterns of health care use. Neurology 58:885-894
5. Lipton RB, Dodick D, Sadovsky R, Kolodner K, Endicott J, Hettiarachchi J, Harrison W (2003) A self-administered screener for migraine in primary care The ID Migraine ${ }^{\mathrm{TM}}$ validation study. Neurology 61:375-382
6. Headache Classification Subcommittee of the International Headache Society (2004) The International Classification of Headache Disorders, 2nd edn. Cephalalgia 24[Suppl 1]:1-160 